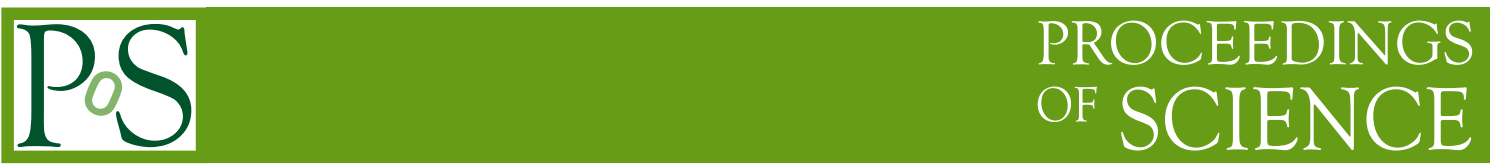

\title{
Neutrino Oscillation Physics at JUNO
}

\author{
Liang Zhan ${ }^{* *}$ \\ Institute of High Energy Physics, Chinese Academy of Sciences, Beijing 100049, China \\ E-mail: zhanleihep.ac.cn
}

The Jiangmen Underground Neutrino Observatory (JUNO) is a multipurpose neutrino experiment being built in China. The primary physics goal is to determine the neutrino mass hierarchy and precisely measure the neutrino oscillation parameters by detecting reactor antineutrinos at a baseline of $53 \mathrm{~km}$ from Taishan and Yangjiang reactor power plants with a nominal thermal power of $36 \mathrm{GW}$. The detector is designed to reach a target mass of $20 \mathrm{kt}$ liquid scintillator and an energy resolution of $3 \% / \sqrt{E}$. JUNO is planning to start data taking around 2020. The principle to determine the mass hierarchy is based on the precision measurement of reactor antineutrino oscillation. The sensitivity of mass hierarchy determination is studied with the standard chi-square fit. The impacts on the mass hierarchy sensitivity from the energy resolution, matter effects, neutron recoiling and other systematic uncertainties are discussed. In addition, the precision of the measurement of neutrino oscillation parameters are studied. Three oscillation parameters, $\Delta m_{21}^{2}$, $\Delta m_{e e}^{2}$ and $\sin ^{2} \theta_{12}$, can be measured with a precision better than $1 \%$.

38th International Conference on High Energy Physics

3-10 August 2016

Chicago, USA

${ }^{*}$ Speaker.

†n behalf of the JUNO Collaboration 


\section{Introduction}

In the three-flavor neutrino oscillation framework, the latest known mixing angle $\theta_{13}$ was found to be about 0.09 which is relatively larger than the expectations before. The precision of $\sin ^{2} 2 \theta_{13}$ can reach to $3 \%$ by continuous running of Daya Bay in the next few years. The next generation neutrino oscillation experiments focus on the determination of the neutrino mass hierarchy (the sign of mass-squared difference, $\Delta m_{31}^{2}$ and $\Delta m_{32}^{2}$ ) and the CP violating phase. Among these experiments, atmospheric [1,2] and accelerator [3, 4, 5] neutrino experiments are proposed to measure the matter effect for the determination of mass hierarchy. Another possibility is to use the oscillation interference effect between $\Delta m_{31}^{2}$ and $\Delta m_{32}^{2}$ by reactor antineutrinos $[6,7,8,9,10]$.

The Jiangmen Underground Neutrino Observatory (JUNO) was proposed [11, 12, 13] to measure the reactor antineutrino spectrum from Taishan and Jiangmen reactor power plants with a $20 \mathrm{kt}$ liquid scintillator (LS) detector. The primary goal is to determine the mass hierarchy and to precisely measure the neutrino oscillation parameters by precise measuring the oscillation spectrum with a $3 \% / \sqrt{E_{v i s}}$ energy resolution. A candidate site is located at Jiangmen in South China, with baselines around $53 \mathrm{~km}$ from Taishan and Yangjiang nuclear power plants. The JUNO experiment has other rich physics potentials, including the detection of supernova neutrinos, geo-neutrinos, solar neutrinos and atmospheric neutrinos, and can probe the sterile neutrinos and exotic physics like nucleon decays.

\section{Mass Hierarchy Determination}

In the propagation of the reactor antineutrinos, the survival probability can be written as

$$
P_{e e}=1-\cos ^{4} \theta_{13} \sin ^{2} 2 \theta_{12} \sin ^{2} \Delta_{21}-\cos ^{2} \theta_{12} \sin ^{2} 2 \theta_{13} \sin ^{2} \Delta_{31}-\sin ^{2} \theta_{12} \sin ^{2} 2 \theta_{13} \sin ^{2} \Delta_{32},(2.1)
$$

where $\Delta_{i j}=1.27 \Delta m_{i j}^{2} L / E, \Delta m_{i j}^{2}$ is the neutrino mass-squared difference $\left(m_{i}^{2}-m_{j}^{2}\right)$ in $\mathrm{eV}^{2}, \theta_{i j}$ is the neutrino mixing angle, $L$ is the baseline length from reactor to detector in meter, and $E$ is the $\bar{v}_{e}$ energy in $\mathrm{MeV}$. In the liquid scintillator detector, the reactor antineutrinos can be detected via coincident signals of inverse $\beta$ decay (IBD) reaction, $\overline{v_{e}}+p \rightarrow e^{+}+n$.

Reactor antineutrinos are mainly generated by Taishan and Yangjiang reactor power plants with a nominal thermal power of $36 \mathrm{GW}$. The baselines and the related reactor power of each reactor core are listed in Table 1. A thermal power of $26.6 \mathrm{GW}$ will be available in 2020 when JUNO

\begin{tabular}{|c|c|c|c|c|c|c|}
\hline Cores & YJ-C1 & YJ-C2 & YJ-C3 & YJ-C4 & YJ-C5 & YJ-C6 \\
\hline Power $(\mathrm{GW})$ & 2.9 & 2.9 & 2.9 & 2.9 & 2.9 & 2.9 \\
\hline Baseline $(\mathrm{km})$ & 52.75 & 52.84 & 52.42 & 52.51 & 52.12 & 52.21 \\
\hline \hline Cores & TS-C1 & TS-C2 & TS-C3 & TS-C4 & DYB & HZ \\
\hline Power $(\mathrm{GW})$ & 4.6 & 4.6 & 4.6 & 4.6 & 17.4 & 17.4 \\
\hline Baseline $(\mathrm{km})$ & 52.76 & 52.63 & 52.32 & 52.20 & 215 & 265 \\
\hline
\end{tabular}

Table 1: List of the baselines and reactor power for the Yangjiang (YJ) and Taishan (TS) reactor power plants, as well as the remote reactors of Daya Bay (DYB) and Huizhou (HZ), at the candidate site. Taken from [13] 
experiment plans to taking data. Remote reactors $(>200 \mathrm{~km})$, such as Daya Bay and Huizhou reactor power plants, give a very small contribution to the total reactor antineutrino spectrum. The experiment site was selected to minimize the baseline difference between Yangjiang and Tainshan reactor cores, optimizing the sensitivity. At the candidate site, the JUNO experiment will collect more than hundred thousand IBD signals over 6 year of data, assuming an $80 \%$ effective detection efficiency which relies on the event selection efficiency, live time ratio and reactor full power ratio.

To study the mass hierarchy sensitivity, we define a standard $\chi^{2}$ function to fit the simulated reactor antineutrino spectrum to the input spectrum in the normal hierarchy $(\mathrm{NH})$ and inverted hierarchy (IH) cases respectively,

$$
\chi^{2}=\sum_{i} \frac{\left(T_{i}-F_{i}\left(1+\varepsilon_{i k}\right)\right)^{2}}{T_{i}}+\sum_{i} \frac{\varepsilon_{i k}^{2}}{\sigma_{k}^{2}},
$$

where $T_{i}$ is the input spectrum assuming the true case is $\mathrm{NH}$ or $\mathrm{IH}$, and $F_{i}$ is the fitted spectrum. The $\varepsilon_{i k}$ is the pull parameters for the systematic uncertainty of $\sigma_{k}$. As expected, the spectrum can fit the input spectrum better if the truth mass hierarchy is used. Assuming $\mathrm{NH}$ is the truth, we try two fits using $\mathrm{NH}$ spectrum and IH spectrum respectively and get two corresponding minimal $\chi^{2}$, namely $\chi_{\min }^{2}(\mathrm{NH})$ and $\chi_{\min }^{2}(\mathrm{IH})$. A discriminator can be defined as

$$
\Delta \chi^{2}=\chi_{\min }^{2}(\mathrm{IH})-\chi_{\min }^{2}(\mathrm{NH}),
$$

A median sensitivity [14], approximatly $\sqrt{\Delta \chi^{2}} \sigma$, is often used when comparing the sensitivity of different experiments.

To optimize the candidate site for JUNO experiment, the ideal case with two reactors was studied. The sensitivity as a function of the baselines of the two reactors is shown in the left panel of Fig. 1. The sensitivity reaches maximal around $50 \mathrm{~km}$, which is approximately at the oscillation maximal baseline for $\Delta m_{21}^{2}$ driven oscillation. The baseline difference of the two reactors is very important as shown in right panel of Fig. 1. If the baseline difference is close to $2 \mathrm{~km}$, where is the baseline for the maximal $\Delta m_{32}^{2}$ driven oscillation, the sensitivity will be eliminated due to the interference of the reactor antineutrino spectra. As a result, the current candidate site is optimized to achieve the equal mean baselines from Taishan power plant and Yangjiang power plant.

Energy resolution is crucial for JUNO experiment because the mass hierarchy sensitivity is determined by the fast oscillation frequency driven by $\Delta m_{32}^{2}$ and $\Delta m_{31}^{2}$, which is about $1 / 30$ of the slow oscillation frequency driven by $\Delta m_{21}^{2}$. The importance of the energy resolution and the event statistics is illustrated in Fig. 2. At the nominal luminosity with an energy resolution of $3 \% / \sqrt{E}$, a sensitivity of $\Delta \chi^{2}=11$ will be obtained. If the energy resolution reaches $2.6 \% / \sqrt{E}$ and $2.3 \% / \sqrt{E}$, a $\Delta \chi^{2}$ sensitivity of 16 and 25 can be achieved, respectively.

The uncertainties on $\sin ^{2} 2 \theta_{13}, \sin ^{2} \theta_{12}$, and $\Delta m_{21}^{2}$ have slight impacts to the sensitivity. However, the uncertainty of effective mass-squared difference $\Delta m_{\mu \mu}^{2}$ ( combination of $\Delta m_{31}^{2}$ and $\Delta m_{32}^{2}$ ) from a prior measurement has large impact to the sensitivity. A sensitivity of $\Delta \chi^{2} \simeq 18$ will be achieved by using a $1 \%$ uncertainty of $\Delta m_{\mu \mu}^{2}$, as shown in Figure 3.

As a summary of the mass hierarchy determination, the impacts of different effects on the sensitivity is listed in Table 2. Matter effects, always treated as a small effect in reactor antineutrino experiments due to low energy, have been considered for the JUNO experiment [15]. A reduction of 0.6 for the mass hierarchy sensitivity is found after considering the matter effects. 

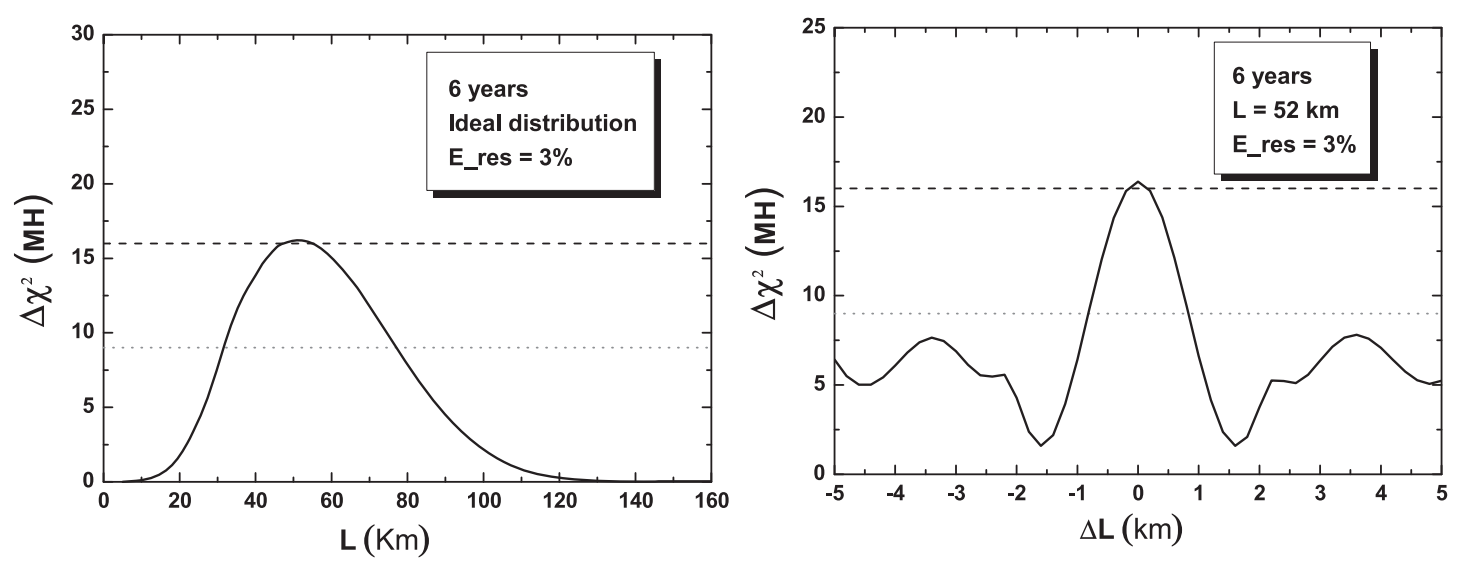

Figure 1: Sensitivity of mass hierarchy determination as the function of the baseline (left panel) and function of the baseline difference of two reactors (right panel). Taken from [13].

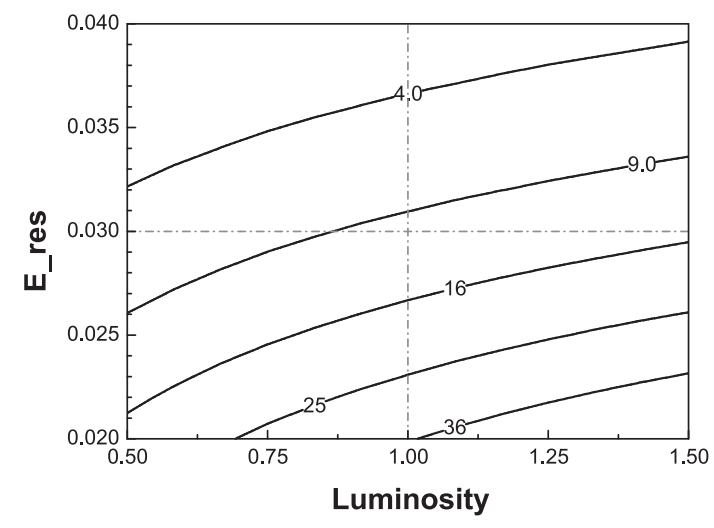

Figure 2: The $\Delta \chi^{2}$ contours as the function of the event statistics (luminosity) and the energy resolution, where the vertical dashedíCdotted line stands for the nominal running of six years with.Taken from [13].

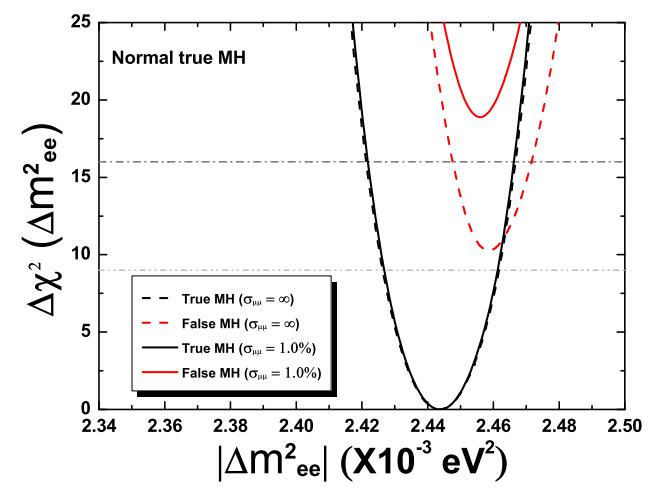

Figure 3: Mass hierarchy sensitivity of JUNO. The solid and dashed lines are for the analyses with and without the prior measurement of $\Delta m_{\mu \mu}^{2}$. Taken from [13].

\section{Precision Measurement of Oscillation Parameters}

Thanks to the large target mass and good energy resolution, JUNO could be the first to observe an oscillation pattern containing two independent oscillation frequencies and multiple oscillation cycles. Therefore JUNO will have an unprecedented precision measurement of $\Delta m_{21}^{2}, \Delta m_{e e}^{2}$ and $\sin ^{2} \theta_{12}$ better than $1 \%$.

The precision of the oscillation parameter measurements were studied using the standard chisquare fit using Eq. 2.2. In this study, effects of important systematic errors, including the bin-tobin (B2B) energy uncorrelated uncertainty, the energy linear scale (EL) uncertainty and the energy non-linear (NL) uncertainty, and background (BG) were taken into account. In Table 3, we show the precision of $\sin ^{2} \theta_{12}, \Delta m_{21}^{2}$ and $\left|\Delta m_{e e}^{2}\right|$ from the nominal setup to those including additional 


\begin{tabular}{l|l|l|l|l|l|l|l}
\hline & Stat. & Core dist. & DYB \& HZ & Shape & B/S (stat.) & B/S (shape) & $\left|\Delta m_{\mu \mu}^{2}\right|$ \\
\hline Size & $52.5 \mathrm{~km}$ & Tab. 1 & Tab. 1 & $1 \%$ & $6.3 \%$ & $0.4 \%$ & $1 \%$ \\
\hline$\Delta \chi_{\mathrm{MH}}^{2}$ & +16 & -3 & -1.7 & -1 & -0.6 & -0.1 & $+(4-12)$ \\
\hline \hline
\end{tabular}

Table 2: Different contributions for the mass hierarchy determination. The first column is the statisticalonly scenario with the equal baseline of $52.5 \mathrm{~km}$, the second column considers the real distribution (dist.) of reactor cores, the third column defines the contribution of remote DYB and HZ nuclear power plants, the fourth column stands for the reduction of the reactor shape uncertainty, the fifth and sixth columns are the contributions of the background statistical and shape uncertainties, the seventh column is the enhanced sensitivity from additional information of $\left|\Delta m_{\mu \mu}^{2}\right|$. Taken from [13]

\begin{tabular}{l|l|l|l|l|l}
\hline \hline & Nominal & + B2B (1\%) & + BG & + EL (1\%) & + NL (1\%) \\
\hline $\sin ^{2} \theta_{12}$ & $0.54 \%$ & $0.60 \%$ & $0.62 \%$ & $0.64 \%$ & $0.67 \%$ \\
\hline$\Delta m_{21}^{2}$ & $0.24 \%$ & $0.27 \%$ & $0.29 \%$ & $0.44 \%$ & $0.59 \%$ \\
\hline$\left|\Delta m_{e e}^{2}\right|$ & $0.27 \%$ & $0.31 \%$ & $0.31 \%$ & $0.35 \%$ & $0.44 \%$ \\
\hline \hline
\end{tabular}

Table 3: Precision of $\sin ^{2} \theta_{12}, \Delta m_{21}^{2}$ and $\left|\Delta m_{e e}^{2}\right|$ from the nominal setup to those including additional systematic uncertainties one by one from left to right column. Taken from [13]

systematic uncertainties one by one. Note the energy-related uncertainties are more important because the sensitivity is mostly from the spectrum distortion due to neutrino oscillations. We can achieve the precision level of $0.5 \%-0.7 \%$ for the three oscillation parameters $\sin ^{2} \theta_{12}, \Delta m_{21}^{2}$ and $\left|\Delta m_{e e}^{2}\right|$. Therefore, precision tests of the unitarity of the lepton mixing matrix is feasible at unprecedented precision levels.

\section{Summary}

The JUNO experiment is aimed at neutrino mass hierarchy determination and precision measurement of the oscillation parameters. Using a $20 \mathrm{kt}$ liquid scintillator target, a $3 \sigma-4 \sigma$ sensitivity after six years of data taking, with a $3 \% / \sqrt{E_{v i s}}$ energy resolution, can be reached. Additionally, three mixing parameters can be measured with a precision better than $1 \%$. The JUNO project was approved in 2013 and is planned to start operation in 2020.

\section{Acknowledgement}

Part of the work in this article is supported by the National Natural Science Foundation of China (11205176), and by the Youth Innovation Promotion Association CAS (2016009).

\section{References}

[1] A. Samanta, Phys. Lett. B 673, 37 (2009).

[2] D. J. Koskinen, Mod. Phys. Lett. A 26, 2899 (2011). 
[3] HyperK Collaboration, (K. Abe et al.), arXiv:1109.3262.

95

[4] LBNE Collaboration, (T. Akiri et al.), arXiv:1110.6249.

96 [5] S. Bertolucci et al., arXiv:1208.0512.

97 [6] S. T. Petcov and M. Piai, Phys. Lett. B 533, 94 (2002).

98 [7] S. Choubey, S. T. Petcov and M. Piai, Phys. Rev. D 68, 113006 (2003).

99 [8] J. Learned, S. T. Dye, S. Pakvasa and R. C. Svoboda, Phys. Rev. D 78, 071302 (2008).

100 [9] L. Zhan, Y. Wang, J. Cao and L. Wen, Phys. Rev. D 78, 111103 (2008).

101 [10] L. Zhan, Y. Wang, J. Cao and L. Wen, Phys. Rev. D 79, 073007 (2009).

102 [11] Y.F. Li, J. Cao, Y. Wang and L. Zhan, Phys.Rev. D 88, 013008 (2013).

[12] Z. Djurcic et al. [JUNO Collaboration], arXiv:1508.07166 [physics.ins-det].

[13] F. An et al. [JUNO Collaboration], J. Phys. G 43 (2016) no.3, 030401 [arXiv:1507.05613 [physics.ins-det]].

[14] M. Blennow, P. Coloma, P. Huber and T. Schwetz, JHEP 1403 (2014) 028

[15] Y. F. Li, Y. Wang and Z. z. Xing, Chin. Phys. C 40 (2016) no.9, 091001 [arXiv:1605.00900 [hep-ph]]. 